\title{
CIENCIA Y VALORES: \\ UN REORDENAMIENTO DE ARGUMENTOS A FAVOR DE LA CIENCIA SOCIAL
}

\author{
Angeles Lizón \\ (Departamento de Sociología de la Universitat \\ Autònoma de Barcelona)
}

Mucho se ha discutido hasta qué punto es necesaria e incluso posible la insistencia de Weber sobre la Wertfreibeit o «imperativo fundamental de la imparcialidad cientificas (1904:49) en las ciencias sociales o de la cultura. En efecto, el esclarecimiento de esta cuestión ha ocupado la reflexión sociológica y filosófica al menos desde Weber (1904) hasta el 15 Congreso de Sociología de Heidelberg (1964) para conmemorar el centenario de su nacimiento.

Las cuestiones planteadas en torno a su propuesta «desvinculación valorativa» o «independencia axiológica» (Wertfreibeit) y la «relación de valor» o «teferencia a valotes» (Wertbeziebung), término este último que emplea para subrayar aquellos criterios necesarios para la selección de datos fácticos en cualquier investigación socio-histórica, han llevado a múltiples reformulaciones de la relación entre ciencias sociales y juicios de valor, ${ }^{2}$

1. Este es un aparte de un trabajo más amplio sobre los debates del método en sociología. Agradezco a Antoni Domènech Figueras, tituiat de Filosofía y Metodología de las Ciencias Sociaies en la Universidad de Barcelona, el haberme proporcionado materiales - suyos y ajenos- así como las conversaciones sobre el tema. Con toda seguridad éstas han contribuido a fijat mi propia posición al respecto.

2. Ct. Topitsch, E., «Max Weber and Sociology Today», pp. 8-26 en Stammer, O. (ed.), Max Weber and Sociology today (1965, alemán), Harper, Tortchbooks, N.Y., 1971. Parsons, T., "Value-freedorn and Objectivity», pp. 27.58, en ibid. Igualmente las discusiones que siguen a la ponencia de Parsons a cargo de Horkheimer, M.; von Wiese, L.; Albert, H.; Habermas, J. y Rossi, P., pp. 51-77. Ver también Albert, H.: «Cono- 
La controversia, en efecto, ha producido tal cantidad de pronunciamientos, muchos de ellos oscuros y otros tantos autocorrectivos y autorrefutatorios, ${ }^{3}$ que se torna difícil el tratamiento del tema. Aquí me ceñiré a otdenar algunas ideas en torno a una simple constatación derivada de la revisión de tales debates: ni las ciencias naturales están stricto sensu libres de evaluaciones, ${ }^{4}$ tesis sobre la que ha faltado el énfasis apropiado entre científcos sociales, ni tampoco las ciencias sociales son necesariamente subjetivizantes y éticamente sesgadas, tesis sobre la que la literatura y práctica sociológica resulta inabarcable.

En cualquier caso, la incidencia de los juicios de valor y prescriptivos en la ciencia no es privativo del ámbito de las sociales; y en relación a estas últimas, puede afirmarse la posibilidad de un tratamiento objetivo de los valores. Ordenar algunos argumentos en torno a esta cuestión es el propósito de los siguientes apartados.

cimiento y decisión", cap. III en su Tratado sobre la razón crítica (1968, alemán), Sur, B. A.; «Social Science and Moral Philosophy", pp. 385-409 en Bunge, M. (ed.), The critical Approach to Science and Philosopby. In bonor of $K$. R. Popper; \&Theotie und Praxis. Max Weber und das problem der Wettkreiheit», pp. 246 y ss. en Oldemeyer, E. (ed.), Die Pbilosophie und die Wissenschatten, Meisenheim, 1967; y junto con Topitsch, L. (eds.), Werturteilstreit, Darnstadt, Wissenschaftliche Bibliothek, 1971. Rossi, P., «Objectivité scientifique et presuppositions axiologiques», en Rev. Int. Sc. Sociales, vol. XVIII, núm. 1, Patís, 1965.

3. A este propósito valdría revisar, por ejemplo, el caso de Gunnar Myrdal, clásico del tema. En su The political element in the development of economic theory, 1929 , sustenta la posibilidad de una ciencia econbmica desinteresada, value-free u objetiva, para pasarse al polo contrario en su revisión del mismo texto (1953: p. vii de la edición inglesa de Routledge, Londres), argumentando la imposibilidad de una «ciencian social. Como abunda en Objetividad en la investigación social (1969, inglés; traducción del Fondo de Cultura Económica, México, 1972, p, 8): «... la ciencin social no es más que sentido común altamente sofisticado", acogiéndose a lo sumo al principio de explicitacion weberiano (Weber, 1904: 47): «... los nedios lógicos disponibles para protegetnos de las desorientaciones son en general éstos: desarrollar una conciencia total de las valoraciones que determinan realmente nuestra investigación teórica y práctica, observat esas valoraciones desde nuestro punto de vista tespecto de la relevancia, significacion y factibilidad en la sociedad estudiada, transformarlas en premisas específicas de valor para la investigación, determinat el enfoque y definir los conceptos en términos de un conjunto de premisas de valor explícitamente asentadas» (ibid., p. 9).

4. Para una crítica a la tesis de la exención de valores en ciencia, cf. Popper, K. R., «Facts, standass and truth: A further criticism of telativism», Addendum (1961) a su The open Society and its enemies, Routledge, Iondres, 1962, 4a. ed., pp. 369396 (traducción castellana de Paidós); «La lógica de las ciencias sociales», Congr. T"ibingen, pp. 101-120, en Adorno, Popper, Dahrendorf et al. (1969, alernán), Grijalbo, 1973. Agassi, Y., «Epistemology as an aid to science», pp. 135 y ss., en Tbe Britisb Journal of Science, vol. X, 1959; Albert, H., 1967, op. cit.; 1968, op, cit.; 1978: Traktat über Rationale Praxis, J. C. B. Moht (Paul Siebeck), Tübingen; así cotno las distintas participaciones en Adorno, Popper, et al. (1969) ya citado. 
Ciencia y valores: tun reordenamiento de argimentos a favor de la ciencia social

\section{FINES Y PROPOSITOS DE LA CIENCIA}

En primer lugar, si bien la ciencia en cuanto tal afirma la objetividad de sus juicios y enunciados (contenidos), tanto las ciencias naturales como las sociales van asociadas a juicios de preferencia, actos de valoración orien. tados a satisfacer ciertos desiderata (verdad, universalidad, simplicidad, univocidad, etc.). Las operaciones fundamentales de descripción, explicación, predicción teórica o previsión tecnológica suponen actos guiados por criterios, algunos de ellos incluso regidos por reglas metódicas referidas a operaciones conceptuales y empíricas que regulan las acciones y operaciones del científico. El lenguaje de toda ciencia --social o natural- ha de incluir, pues, enunciados valorativos que expresan estos juicios de preferencia. No obstante, dichos critetios epistémicos y metódicos no tienen por qué influir en el contenido objetivo e informativo de los resultados, aunque ciertamente regulan su obtención.

En la discusión ciencia-valores, esto lleva a una primera distinción entre valoraciones en general y evaluaciones estrictamente éticas. Como acaba de afrmarse, el lenguaje de la ciencia incluye juicios de preferencia o evaluativos, acumulados en las tradiciones científicas y sometidos a cribas específicas, muchas de ellas reguladas e institucionalizadas a través de la propia comunidad científica. Cabe, no obstante, apuntar al hecho de que el conjunto de los enunciados evaluativos (valoraciones y prescripciones) es necesariamente más amplio que el de los juicios morales o éticos. En este último caso, los juicios éticos son sólo un subconjunto de los juicios evaluativos. Ello permite una primera distinción analítica entre juicios no-éticos (criterios autorregulativos) incorporados al lenguaje de la ciencia -tanto social como natural-y juicios éticos (que implican consideraciones personales) que notmalmente acompañan a la actividad científica, ya sea individual o institucionalmente organizada y regimentada. ${ }^{5}$

Popper ${ }^{6}$ sisternatiza esta diferencia estableciendo la distinción entre valores estrictamente científicos, problemas concernientes a toda una familia de intereses regulativos vinculados a la validez y certeza, a la tele-

5. Pata una crítica del lenguaje y el dualismo conocimiento/decisión, ct. Albert, 1968, op. cit., particularmente su capítulo III; a este respecto ver los útiles comentarios de Muguesza, J. a R. S. Rudner, «Etica y Ciencias sociales», pp. 231-274 en Filosolia y ciencia en el pensamiento español contemporáneo: (1960-1970), Simposio de Lógica y Filosofía de la Ciencia, Ed. Tecnos, Madrid, 1971. El propio Rudner, «The scientist qua scientist wakes value judgementsm, Pbilosophy of Science, 20.1, 1953; Stevenson, Ch. L., Ethics and Language, New Haven, 1944 y Bunge, M., Etica y Ciencia, Siglo XX eds., B. A., 1960.

6. Popper, K. R. op. cit, en Adorno, Poper et al. (1969, alemán), La disputa del positivismo en la sociología alemana, Grijalbo, 1973. 
vancia, simplicidad, sistematicidad (el «interés científico por la verdad»), por un lado, y valores e intereses extracientíficos que concurren en el hacerse de la ciencía, por otro (1969: 93). Con ello, más que exigir o pautar la desvinculación valorativa, lo que hace es establecer la diferencia entre distintas esferas de valor $\mathrm{y}$ atribuirles diferentes ámbitos de incidencia (ibid.: 111). Así, se puede sostener que, por una parte, la objetividad en ciencia no es asunto de ética personal (la objetividad de la ciencia no depende de la objetividad del científico: 109), sino que es un asunto (de la comunidad científica) de crítica objetiva tecíproca (110); y, por otra, afitmar que es imposible etradicar los valotes extracientíficos del quehacer de la ciencia. Privado de estos valores, el científco, como cualquier otro hombre carente de ellos, se empobrecería: «EI científico, objetivo y "libre de valores" no es el científico ideal. Sin pasión la cosa no matcha, ni siquiera en la ciencia pura» (111). «Descaractetización» y «objetividad» científica no tienen nada en común, como diría Weber (1904:49).

La Wertfreibeit se interpreta así como un criterio metódico que, en tanto tal, conlleva una función normativa limitada por sus propios principios autorregulativos. La exención de valor hace referencia a criterios lógicos, epistémicos y prácticos que validan y confirman los enunciados 0 sistemas de enunciados de naturaleza cognitivo-informativa, en tanto tesultados del trabajo conceptual sistemático sobre la realidad (ciencia producida). Este comportamiento humano «desinteresado» se sustenta sobre «determinados estándazes» y se «aproxima a determinados ideales», por lo que considera sus productos como «válidos, fácticamente dependientes del hecho de que idealizan determinadas condiciones cuya no existencia tendría como consecuencia un curso distinto de esta actividad y, en general, otros resultados»?

Habría que precisar algo más algunas cuestiones referentes a los valores cientificos o «criterios internos» autorregulativos. Los enunciados y sistemas de enunciados de la ciencia empírica son asertorios e informativos. Afirman algo sobre cosas, informan sobre cosas. Su coherencia interna y su correspondencia externa (elementos de juicio sintácticos y semánticos) permiten evaluar los grados de certeza y la pertinencia informativa de los mismos. Su grado de objetividad, en este sentido, depende de reglas lógicodeductivas y criterios de validación fáctica o contrastación. No obstante, es evidente que, so pena de circularidad viciosa, las tesis to los sistemas de tesis), aceptadas por aplicación de criterios epistémicos específicos conside-

7. Albert, 1968, op. cit., particularmente, pp. 94 y ss, donde formula sa posicion frente a la cuestión de exención de valores. Cf. también su 1967 en Oldemeyer (ed.), op. cit. 
Ciencia y valores: un reordenamiento de argumentos a favor de Ia ciencia socia:

rados apropiados, no pueden ser evaluadas como productos construidos (o. tesis fácticas) sólo en función de aquellos mismos criterios que llevan a su consecución. La elección entre dos hipótesis diferentes (igualmente verdaderas o plausibles) sobte $(x)$, que utilizan los mismos criterios epistémi$\cos (y)$, no puede realizarse sólo en función de $(y) .^{8} \mathrm{La}$ introducción de ciertos criterios prácticos (potencia informativa, control aplicativo y eficacia práctica) constituye una segunda categoría de controles racionales sobre los productos cientificos.

La «bondad» epistémica de las tesis aceptadas viene también así justiffcada por referencia a ciertos criterios prácticos, idea que está en fundamento del pragmatismo metodológico moderno. En su Sistematización Cognoscitiva, Rescher (109 y 116-117) hace refetencia al éxito de las predicciones y aplicaciones y a la eficacia de las tesis asertadas. Las teorías empiricas no están interesadas, $\mathrm{o}$ al menos no simplemente, en la construcción de modelos o sistemas conceptuales consistentes. Al pretender un conocimiento fáctico, los esfuetzos científicos se orientan a la búsqueda o acopio de información correcta sobre el mundo (la reducción de la ignorancia de la que habla Popper: ibid., 101 y ss.), por lo que su progreso se puede visualizar como el creciente acúmulo de información teóricamente sistematizada que Uleva a la progresiva solución de problemas y al control tanto cognoscitivo cuanto físico de los mismos (Rescher, 1978).

Vearnos ahora: la introducción de estos criterios prácticos, ¿no plantea una discordancia de objetivos? ¿Altera o no la supuesta «objetividad» de los contenidos de la ciencia? Por supuesto que los distintos objetivos no han de ser necesariamente coincidentes, ni tampoco han de concurrir en el mismo grado (como diría Pareto, una teoría puede, por ejemplo, ser verdadera sin ser útil, y útil sin ser verdadera), y está claro, también que las. distintas corrientes de pensamiento han propuesto diferentes objetivos a la ciencia. Pero ésta es una cuestión que aquí se dejará de lado. Lo que se pretende afirmar es algo más específico y tiene relación con el segundo interrogante.

Si la objetividad de una tesis fáctica (y, en general, del conocimiento científico) viene garantizada por el método de construcción utilizado para su aserción (cf. Popper, 1969:110), la justificación de este método de construcción (o de los criterios de procedimiento sistemático y contrastable) se evalúa mediante criterios prácticos y a partir de la efectiva adecuación

8. Rescher, N., 1979 (inglés), Siglo XXI eds., México, 1981, en su argumentación a favor de la introducción de estos nuevos criterios pragmáticos (criterio de eficacia) apela al «argumento de la rueda» de Montaigne y a su requerimiento de un instrument judicatoire o método de decisión adicional. Ver capitulo IV, particularmente, pp. 106-109. 
de sus resultados o de la eficaz resolución de los problemas que se plantea. En la versión pragmática, la legitimación de los contenidos de la ciencia (controles teóricos y controles operativos de aplicación) se justifica o valida, en última instancia, en el ámbito de sus aplicaciones (Rescher, 1979: 106-109).

Estos criterios evaluativos (epistémicos y prácticos) constituyen mecanismos operativos internos de legitimación de las tesis fácticas y -en tanto principios regulativos de validez y pertinencia cognoscitiva de la ciencia-no evalúan o justifican el uso o la utilización de sus predicciones o previsiones ni de sus propias derivaciones (aplicaciones) tecnológicas. Son critetios autorregulativos internos y según ellos sólo se confiere «bondad» epistémica a las teorizaciones científicas.

\section{CIENCIA Y ÉTICA DE LA RESPONSABILIDAD}

Si lo afirmado acerca de las valoraciones epistémicas y prácticas que sustentan los objetivos y logros de la ciencia admite la incorporación del principio de objetividad crítica en su concepción metodológica de los contextos validatorios de teorías (productos científicos), ello no justifica su simple extrapolación a los demás contextos de la ciencia. Una cosa son las razones que sustentan una teoría, otra el proceso de investigación y cons. trucción de teorías y, aún otra más, la aplicación tecnológica de sus detivaciones.

Por su parte, la ciencia, además de un lenguaje o sistema conceptual bien construido y validado, es una compleja actividad humana y, en tanto artefacto construido por hombres, ésta no puede agotarse en su propio contenido. Por lo que una consideración sobte las evaluaciones (valotes y normas) en ciencia plantea, al menos en principio, una definición y una vinculación diferentes ya sea que los juicios evaluativos se tefieran al dominio de los productos o tesis fácticas sustentadas (la legitimación de la «ciencia hecha»), o al ámbito de las acciones vinculadas al ptoceso mismo de producción de ciencia, al proceso de su confección y al de su utilización o aplicación, tespectivamente.

Son varias las acciones que concurten en el trabajo científico y que pueden vincularse a los enunciados o sistemas de enunciados que sustenta la ciencia: la elección de problemas, la preferencia de líneas de investigación, la selección de resultados, Ia determinación de rutas de hallazgos, la publicidad de sus conclusiones, la puesta a prueba o experimentación, todas ellas son acciones que concurren en el hacerse de la ciencia, acciones vincu- 
Ciencia y valores: un reordenamiento de argumentos a favot de la ciencia social

ladas a la descripción o explicación científicas y a las aplicaciones derivables de las sistematizaciones teóricas. ¿Qué decir, pues, de estas acciones virculadas a la ciencia?

Al igual que cualquier otro tipo de comportamiento, estas acciones vinculadas a la ciencia son atribuibles a agentes particulares (individuos $O$ grupos) y vienen regidas por criterios prácticos. Como desde Max Weber y su noción de «ética de la responsabilidad» han afirmado tantos otros autores, aquí el compromiso ético no es tanto un hecho cuanto un deber. Puesto que en la acción se da siempre una intención, cualqujer acción que a alguien resulte contraria a sus principios y valores es nociva (contraria a tales valotes) así se haga en aras de la ciencia pura. El propósito (fin) explícito que están llamadas a satisfacer dichas acciones pasa a ser constitutivo de las mismas. Por esta razón pueden (y deben) set examinadas normativa e intencionalmente como cualquier otro tipo de comportamiento o acción.

$\mathrm{Y}$ es aquí, precisamente, donde se plantea a quienes ejercen la ciencia (al actor social), a título personal o colectivo, el problema de la opción moral. Los juicios evaluativos propiamente éticos en materia de ciencia (social o no) - tal es la sugestiva tesis de Amartya Sen en Accounts, Actions and Values - 9 conciernen necesariamente a las actividades relacionadas con la ciencia y no (o al menos no necesariamente) a sus productos. Así, una cosa es, por ejemplo, el juicio evaluativo de pertinencia informativa aplicable a las tesis fácticas, relacionado a lo que Amartya Sen llama su interés de uso informativo, y, otra, el juicio valorativo de las acciones vinculadas a la construcción y aplicación de la ciencia. Éstas (las acciones vincu. ladas al producto científico) quedan intrínsecamente supeditadas al interés de uso de la información para todas las partes interesadas (promototes, fi" nanciadores, investigadores y receptores), e incluyen propósitos y valores prácticos, requerimientos individuales y sociales no-teóricos que escapan al propósito sistemático e informativo de los contenidos científ́cos.

En tanto incorporan la intencionalidad y' propósito de sus géstores, las acciones vinculadas a la ciencia teórica y sobre todo a sus aplicaciones, directa o indirectamente afectan a todas las partes involucradas en su utilización y, ésta, ciertamente, no sirve a todos por igual. ${ }^{10}$ En este sentido, la

9. En realidad, ésta es la tesis del racionalismo crítico. $C t$., por ejemplo, Albert, 1968. La referencia de A. S. en pp. 87-107 de Lloyd, Ch. (ed.), Social Tbeory and Political practice, Walfson College Lectures, 1981, Claredon Press, Oxford.

10. Jugando con la idea de teorias interesantes para fines de conocimiento que expresa en su noción de use interest, en tanto referida a tesis fácticas y a acciones vinculadas a ellas, Amartya Sen establece la diferencia entre el use-interest relative (atinente a la evaluación de los enunciados y sistemas de enunciados pivotados sobre sus propios contenidos cognitivo-informativos) para los distintos grupos interesados en la 
creciente aplicación tecnológica derivada de la ciencia teótica moderna ha explicitado la pérdida de equilibrio entre el riesgo teórico de refutación (que atañe a la ética del conocimiento) y el riesgo práctico (que implica a la ética de la responsabilidad). Con ello se plantean cada vez más (y más graves) problemas estrictamente éticos al colectivo científico.

Aunque es evidente que la cuestión alcanza televancia en las dos últi. mas décadas, la preocupación por los problemas morales de la ciencia no es, precisamente, un fenómeno reciente. Es verdad que, en términos genetales, los filósofos y científicos de orientación analítica soslayaron las cues. tiones éticas por considerarlas inabordables desde la perspectiva lógica de la ética del conocimiento. Pero el interés por los problemas de la conducta ética de la ciencia está ya presente a principios de siglo, claramente a partir de la crisis de conciencia que sigue a la primera conflagración mundial.

Está por demás decir que las dificultades de concordancia entre la ética del conocimiento y la ética de la tesponsabilidad, entre conocimiento y consecuencias prácticas del conocimiento, quedan cada vez más explicitadas en nuestra cultura científica de finales de siglo. No obstante, dada la intención específica de este ejercicio, dejaré de lado esta importante cuestión ${ }^{11}$ seña*

información, y el everytbing considered vinculado necesariamente a la evaluación de las acciones que acompañan a la ciencia. La ilustración que ofrece (pp. 104106) para establecer la diferente naturaleza de estas dos evaluaciones (la de las tesis fácticas, por una parte, y la de las acciones conexas, por otra) resulta clarificadora. Tomando un programa de la BBC sobre muerte cerebral y utilización clínica de órganos, emitido en octubre de 1980 , y prescindiendo del aspecto técnico (que escapa tanto a su como a nuestro tema), combina el critexio de use interest sobre las dos coordenadas básicas de su trabajo.

Respecto a la tesis fáctica (informe, descripción, etc,), el juicio evaluativo (acerca de su 'bondad' o no) tiene relación con los diferentes intereses de uso que sirve. En el caso mencionado, el reporte sirve bien los intereses informativos de los médicos especialistas que a partir de entonces tomarán las precauciones clínicas y legales pertinentes. Sirve, en cambio, mal a los potenciales donantes de órganos, innecesariamente atemo. rizados ante la información; $y$, simplemente deja fuera del interés informativo a grupos directamente afectados (el de los enfermos renales en espera de transplante) cuyo interés próctico es la obtención de órganos. Tomando en considetación todos los as. pectos, e incluyendo a todos los grupos afectados por el informe, cabe preguntarse si Ia acción de darle publicidad puede evaluarse como 'buena', aunque la información en sí sea correcta. El ejemplo resulta quizá demasiado sencillo (todos los que utiliza en este ensayo lo son), pero sirve para iluminar la tesis básica: «Moral judgements in the fields of science - social and natural-directly concern actions, not accounts", p. 107.

11. Ct. Albert, 1968: «Racionalidad y Compromiso», pp. 9 y ss. Pata una recien. te exposición que abunda en el tema y hace referencia directa a la contraposición entre ética del conocimiento y ética de la tesponsabilidad, of. A. Domènech F., «La ciencia moderna, los peligros antropogénicos y la racionalidad de la política de la cien. cia y de la técnica", pp. 9-52, en Arbor, nứm. 481, enero, 1986. 
lando, en cambio, que ello no tiene por qué llevar a la confusión de planos. Como en términos bastante felices enunciaba M. Sacristán (1981): «... precisamente la peligrosidad o "maldad" práctica de la ciencia contemporánea es función de su bondad epistemológica.» ${ }^{12}$ La posibilidad aplicativa de la ciencia teórica (sus posibles detivaciones tecnológicas) es a lo sumo una condición de su valor epistemológico (aplicabilidad en principio), y ha de distinguirse de su uso o aplicación efectivos. La aplicación de la ciencia empírica teórica y de sus derivaciones implica, además, una decisión o un propósito extracientíficos y constituye una acción (individual o colectiva) sobreañadida a la de su «buena» confección.

Habria que establecer, pues, una distinción entre, por una parte, la exactitud de una teorización científica, su valor cognitivo-informativo y bondad epistemológica y, por otra, la aptitud o propiedad (no-contradictoriedad medios-fines) de la(s) acción(es) vinculada(s) a la ciencia. En el primer sentido resulta cuestionable - al menos en principio y cualquiera que sean las especificidades técnico-metódicas de cada una de ellas- que el científico de la naturaleza esté más libre de elementos evaluativos que el científico social. Para ambos resulta inevitable la presencia de criterios metodológicos (lógicos, epistémicos, fácticos y pragmáticos) en ciencia. Y si según los estándares admitidos y ya clásicos de la metodología científica la presencia de estas evaluaciones no-éticas en la ciencia «hecha» deja intacta la «objetividad» de las hipótesis y teorías de las ciencias naturales, la «cientificidad» de las hipótesis sociológicas no tendría por qué verse sobrecargada, o al menos no en este sentido. En ambos campos resulta evidente la ineludible importancia del recurso a la lógica y la experiencia, y en ambos casos los científicos se hallan reducidos -en tanto tales- a la exactitud y eficacia de sus informaciones (ética del conocimiento). Respecto a la segunda cuestión, actividad cientifica y conducta moral no son independientes (ética de la responsabilidad) y ello, por supuesto, tampoco es exclusivo de las ciencias sociales.

\section{CIENCIA SOCIAL Y VALORES}

Una vez admitido que la praxis de la ciencia no está libre de valoraciones éticas, habría que señalar que una cosa es la evaluación de las accio-

12. «La telación entre la sociedad y la naturaleza en la filosofía de las ciencias sociales* (Un esquema a discusión). Comunicación presentada en el Congreso Mexicano de Filosofía (Guanajuato, diciembre 1981), en Mientras tanto, núm. 10, diciembre 1981. 
nes vinculadas a la ciencia que dan cabida a su enjuiciamiento propiamente moral, y, otra, si la teoría incorpora (o no) valotes o notmas y si puede sostenetse independientemente de ellos.

Dejando de lado la vertiente estrictamente normativa (tanto las ciencias sociales como las ciencias naturales admiten la distinción entre ciencias normativas y ciencias fácticas), paso directamente a la cuestión, tantas veces debatida, de la posibilidad de estudio sistemático y objetivo de los valores. Por supuesto que las ciencias formales y, ciertamente, las ciencias traturales modernas no se ocupan de valores. En abierto contraste, las ciencias bumanas y de la cultura los tienen por objeto de reflexión y análisis. Precisamente uno de los argumentos recurrentes contra la posibilidad de otorgar estatuto científico a estas ramas del conocimiento ha sido la naturaleza específica de su objeto de estudio (los valores) y su supuesta irreductibilidad a explicaciones por causas materiales.

La mayor parte de las confusiones a este respecto provienen de la no distinción de los planos en que se sitúa la dicotomía hecho-valor. Debería, por lo tanto, aclararse ante todo esta cuestión fundamental. Si bien lógica y conceptualmente los valores no son deducibles de los hechos (desde Hume sabemos que el deber ser no se sigue logicamente del ser), ontológicamente, en cambio, sí que pueden ser reducidos a ellos. Separados en el nivel lógico, hechos y valores se unen en el plano ontológico. Como sefrala Bunge (1960: 30-31), los valores son sólo «... propiedades relacionales que adjudicamos en ciertas ocasiones a cosas, actos o ideas en relación a determinados desiderata (...). No constituyen, pues, un mundo aparte del mundo de las cosas, actos o sistemas conceptuales que juzgamos valiosos, del mismo modo que los colores no constituyen una esfera aparte de los objetos coloreados. Los valores no se oponen a la realidad, sino que son un aspecto de ese trozo de realidad elabotada por nosotros a la que Ilamamos experiencia». Pueden, pues, ser considerados como «relativos» a hechos, códigos, sucesos, comportamientos o acciones individuales o colectivas y, en tanto tal, ha de ser legítimo su tratamiento objetivo.

Es verdad que la objetivación de valores y propósitos presenta dificultades de procedimiento que dejaremos de lado. ${ }^{13}$ Valga con apuntar al hecho de que los valores en tanto estados subjetivos o disposiciones ${ }^{14}$ plan-

13. Para una buena revisión ya clásica de las dificultades de procedimiento especificas de las ciencias sociales, ct. Nagel, E., La estructura de la Ciencia. Problemas de la logica de la investigación científica. Edición casteilana de Paidos, 3a., 1978, particularmente el capítulo XIII: «Problemas metodológicos de las ciencias soxiales», pp. $404-452$.

14. Adherirse a ciertos valores equivale açuí a estar en tn cierto estado subjetivo que se expresa en posibles conductas manifiestas y objetivales. 
Ciencia y valores: un reotdenamiento de argumentos a favor de ta ciencia social

tean problemas relativos a la cantidad de elementos de juicio confirmatorios que requieren los enunciados introspectivos para su aceptación. Pero ésta, aunque ciertamente con grados de complejidad diferenciada, es también una situación habitual en las ciencias naturales. Incluso en sus teorías más fuertes, los disposicionales se sustentan sobre un mínimo de elementos de juicio. Por lo demás, la ciencia tampoco trabaja con «hechos puros», sino con hechos interpretados en y para una teoría y, en este caso, la diferenciación entre "carga teórica» y «carga empática» (interpretativa o comprensiva) parece diluirse a la hora de evaluar el sentido y la función epistemológica de los términos con que trabajan las distintas ciencias.

Lo hasta aquí afirmado permite dos sentidos diferentes de «valor» ya recogidos en las nociones weberianas de «juicio de hecho» y «juicio de valor», y que suponen la diferenciación entre el juicio evaluativo que expresa aprobación o desaprobación de ciertos ideales motales o no (lo que Beltrán ${ }^{\text {is }}$ enuncia como valores "valiendo») y el sentido en el cual el mismo juicio de valor se trata como un estado subjetivo que se expresa en conductas objetivables (o cuanto valores «siendo»). Esta últina es la noción que está en el fundamento de la crítica técnica. Propuesta inicialmente por Weber (1904: 42 y ss.), dentro de la corriente filosófica analítica interesada en las ciencias sociales, es sistematizada por $K$. $R$. Popper y desarrollada posteriormente por otros flósofos y científicos sociales. Es particularmente H. Albert quien avanza en la propuesta popperiana de una tecnología social como fundamento de la acción social tacional. ${ }^{16}$

La crítica técnica orientada al pronóstico y al examen de la adecuación de medios a fines, a la obtención de las consecuencias buscadas, y a una adecuada relación coste-beneficio en su satisfacción, cuaja en la lógica situacional como método de "comprensión objetiva» (Popper, 1969: 117119). Esta última, consistente en la reducción de las condiciones subjetivas psicologizantes a momentos objetivos de la situación, hace hincapié en el carácter individual de la acción («las instituciones no operan; actúan los individuos en y para las instituciones»: ibid,, 119) y, además, en la objetivación de los elementos psicológicos (lo que le permite deslindar los

15. Beltrán, Miguel, «Acerca de Weber y su ciencia exenta de valoraciones», pp. 397-421, en Jiménez Blanco, J. y Moya, V. C. (dirección y prólogo), Teoría sociológica contemporánea, Ed. Tecnos, Madrid, 1978.

16. Ct. particularmente Popper, 1957 (inglés), La miseria del bistoricismo, Taurus 1961, Alianza 1973, donde introduce abiertamente la idea; asimismo, Popper en Adorno, Popper, 1969, donde la sistematiza. Para ulteriotes desarrollos, cf. Albert, 1968 y 1978, así como los debates sobre el tema compilados en Adorno, Popper, 1969, pp. 147.312. Para el esclatecimiento de la posición de Habetmas (su contendiente intelectual en estos debates), cf. así mismo Habermas, 1963 (alemán), Theorie und Praxis, parcialmente traducido. Editora Sur, B. A., 1972. 
campos de la sociología y la psicología: 116-117), apuntando así a una explicación de las acciones sociales en términos de mecanismos causales intencionales.

La situación viene descrita como el conjunto de recursos y constricciones presentes, el contexto de interacción (que, obviamente, incorpora a otros sujetos intencionales igualmente activos) y de ptesión ejercida (e internalizada) por la vida institucionalmente regulada (teoría de las instituciones: 119). Tal objetivación de los elementos ptesentes en la situación permite reconstrucciones inteligibles a partir de las cuales se organizan los universos de observación e interpretación intencional de los comportamientos individuales socialmente contextualizados y da cabida a una explicación racional de la acción social.

En este último sentido, dado que el criterio de explicación causal verdadeta constituye, desde el punto de vista del falsacionismo, el principio regulativo del procedimiento «científico ${ }^{17} \mathrm{y}$ del resultado «objetivo» de toda ciencia, ${ }^{18}$ el estudio de los valores puede alcanzar tal estatuto de objetividad científica (ibid., 117-119). La evaluación de los propios valores escapa, en cambio, a las posibilidades de este esquema interpretativo de la acción social. En este sentido, afirma Albert, la función de la ciencia sacial es sólo «... investigar las posibilidades prácticas y (...) averiguar con ello cómo podemos dominar la situación existente» (1968: 100); pero este análisis -en el mejor sentido weberiano- «... no nos dice que podemos realizar una de las posibilidades que están en consideración, ni nos prescribe nuestra decisión” (ibid.).

EI trabajo sobre la objetivación y la sistemática de valores ha sido una preocupación constante en la sociología moderna, tanto de corte operacio-

17. El esquema lógico consiste en una inferencia deductiva que parte de teotías (conjeturas) y da unas condiciones iniciales. Si las premisas son verdaderas, lógicamente la conclusión (solución) deberá serlo. Si la conclusión es falsa, al menos una de Ias premisas es falsa [la «teoría de la transferencia de la verdad de las premisas a la conciusión (...) e, inversamente, la teoría de la tetransferencia de la falsedad de la conclusión a por lo menos una de las premisas» (1969, tesis XVII: 113)]. Desde el punto de vista del falsacionismo, «la lógica deductiva queda convertida en el criterio metódico de racionalidad críticas (tesis XVIII: 113).

18. «La llamada objetividad de la ciencia radica en la objetividad del método crítico, Su argumentación general viene compendiada en los 6 puntos de su tesis principal (tesis VI, pp. 103-104). Una revisión de sus posiciones y de los argumentos que fundamentan su propuesta de la logica situacional como salida analítico-sistemática a las ciencias de vaiores (a la sociología en particular) exigiría un trabajo aquí no contemplado. Cf., sus principales contribuciones: 1934: The logic of scientific discovery, Hutchinson y Co. Ltd, Londres, 1953 (traducción castellana de Tecnos); 1965 : Conjeturas y rejutaciones (inglés), Paidós, 2a, ed., 1979; 1972: Conocimiento objetivo (inglés), Tecnos, 1974. 
Ciencia y valotes: un reordenamiento de atgumentos a favor de la ciencia social

nalista (P. F. Lazarsfeld y su noción de estructura latente, disposición o variable intermedia ${ }^{19}$ lo testimonia en el caso del survey analysis), como - así lo hemos visto- de la sociología comprensiva de origen weberiano. Para la sociología inductivo-estadística, si bien el paradigma de la acción ha funcionado como fecundo integrador y la afnación de sus instrumentos bá sicos ha ciertamente instado al rigor sistemático, quedan fuera demasiadas cuestiones que competen a la sociología. La versión inductiva de la acción social se debilita a finales de los años cincuenta al constatat sus propios límites, que Oberschall (1979) sintetiza particularmente en dos: ante todo, la ausencia en ella de un modelo de elección o decisión racional, lo que la reduce al simple tratamiento inductivo de regularidades empíricas en condiciones de reiterabilidad y multiplicidad de procesos. Por otra parte, la constatación de la ineficacia de su propio instrumento matemático, o el límite del análisis factorial en casos de cortelación que incorporan variables individuales y colectivas indistintamente.

Por su parte, el resurgimiento de la sociología comprensiva de origen weberiano, de la crítica técnica orientada a la adecuación de medios-fines mediante el recurso a la explicación intencional, se asocia con nuevos intentos de mrodelización matemática y se apoya en los avances logrados, particularmente en el campo de la psicología experimental y en el del análisis microeconómico, mediante su utilización. ${ }^{20}$ Empieza así a imponerse la superación de la teoría empírica de la acción mediante una lógica de la acción social sustentada en modelos más apropiados de decisión y elección racional. Una lógica de la acción individual, de la decisión individual o de la conducta individual (Boudon, 1984) que, en contraste con el sociologismo (el individuo como fruto de las estructuras sociales) y el atomismo (de los simples agregados de individuos), se sustente en modelos para los cuales

19. Son todas nociones que Lazarsfeld trabaja como pertenecientes a una misma categoría l6gica y operantes en un mismo plano epistemológico. Tales nociones aparecen directamente vinculadas a su trabajo sobre el lenguaje indicial y a su teoría inductivo-estadística de la acción social. Cf. Lazarsfeld, 1970, Philosopbie des social sciences, Paris, Gallimard; Qualitative Analysis: Historical and critical essays, Boston, Allyn y Bacon, 1972; y su coedición con Rosenberg, 1955 (inglés), recogida en su casi totalidad en la edición francesa de Boudon y Lazarsfeld, 1965, 1966 y 1970 (francés), traducida por Laia, 1973,74 y 75 , respectivamente.

20. Hoy los modelos estructurales y analítico-interpretativos (Rapport, A., 1983, Matbematical models in the social and behavioral Sciences, John Wiley e hijos Inc.) ofrecen un nuevo instrumental matemático para la ejexcitación y desarrollo del paradigma de la acción individual socialmente contextualizada (Goldenberg y Duncan [eds.], Structural equation models in the social sciences, N. Y. Seminar Press, 1973) y constituyen un salto en calidad en el tránsito de la teoría empírica (estadística) de la acción a la lógica de la acción social. 
los actores, como variables, ${ }^{21}$ dependen del contexto social. A diferencia de una visión determinista (à la Spencer) del bomo sociologicus, «vinculada a una estrecha concepción positivista de la sociología» (Boudon, 1979), se propone como programa una lógica de lo social que analice la acción individual dentro del contexto social y los efectos de la estructura social sobre la misma. ${ }^{22}$

\section{VALORES Y CIENCIA SOCIAL}

Hay al menos otro sentido en el cual los valores pueden constituir materia de discusión en términos de objetividad y ciencias sociales. A diferencia de los valores objetivables sobre los que se ha propuesto la crítica técnica, los valores también pueden ser considerados en un sentido weberiano como criterio metodológico de selección o delimitación de objetos de investigación. La relación a valores puede aquí claramente operar como criterio instrumental de conformación de unidades significativas e inteligibles (es decir, comprensibles) y cognoscitivamente sistematizables (Weber, 1906: 134 y ss.; 1917: 246). En este sentido la Wertbeziebung funciona como principio heurístico $\mathrm{y}$, en tanto tal, remite al origen de las hipótesis del trabajo investigativo y sistemático.

Los contextos heurísticos, en gran medida patrimonio de todos y tierra de nadie, han sido objeto de múltiples debates de filósofos e historiadores de la ciencia (sociólogos más o menos orientados como A. Gouldner incluidos), pero su tratamiento dista mucho de ser sistemático y su exploración queda fuera de las expectativas de este ejercicio. No obstante, y a favor de la argumentación weberiana, cabría señalar que el recurso a delimitaciones significativas desde perspectivas culturales compartidas (micro o macroculturas), la "concepción deI mundo», el point of view o la Weltanscbauung de los filósofos e historiadores de la ciencia, ${ }^{23}$ es por lo demás

21. Coleman, J. S., «Purposive actors and the mutual effects», pp. 98-118, en Merton, Coleman y Rossi (eds.), Qualitative and quantitative social research. Papers in bonor of Paul F. Lazarsfeld, The free Press, MacMillan, publ. Co. Inc., 1979.

22. Boudon, R., 1979 (francés), La lógica de lo social. Introducción al análisis sociológico, Rialp, 1981; «L'individualisme methodologique en sociologie», pp. 268277, en Comentaire, París 7, núm. 26, 1984.

23. Aparte naturalmente de sus propios introductores, filósofos e historiadores de la ciencia (Koyré, Kuhn, Toulmin, etc.), que sería inútil citar aquí, puede encontrarse una revisión compendiada de estas nociones y su influencia en el panorama de la teoría de la ciencia, en Suppe, F., 1974 (inglés), La estructura de las teorias cientificas, Editora Nacional, Madrid, 1977, cap. V. B., pp. 155-255; y en Shapere, D., 
un hecho más que recurrente en la ciencia en general. $\mathrm{El}$ conjunto de preguntas o problemas formulados en la ciencia empírica (incluida la ciencia «dura») se sustenta sobre supuestos no sólo lógico-matemáticos sino también metafísicos, admitidos por las comunidades científicas. Lo que $N$. Rescher (1979) enuncia como la «disponibilidad de preguntas en la investigación empírica» (p. 148), el «estado de cuestionamiento» del momento (p. 155), o la «especificación del foco problemático» (p. 122; por supuesto que Rescher no se refiere a las ciencias sociales). Si algún efecto claro tuvo La estructura de las Revoluciones científicas de T. S. Kuhn, fue precisamente el de estandarizar y extender esta idea a toda teoría científica. ${ }^{24}$

En este sentido, como en su artículo de 1973 enunciaba de pasada el sociólogo S. Giner, no es «... nada obvio que las orientaciones de valor pesen más en las ciencias humanas que en las demás», aunque, y esto hay que reconocerlo, una vez iniciado el procedimiento de elaboración investigativa o sistemática, «... las ciencias sociales poseen mayores servidumbres». ${ }^{25}$ Es evidente que factores como la naturalidad, proximidad, cotidianidad y familiaridad humana con sus propios dominios, con la propia experiencia condensada en lo que genéricamente llamamos la «cultura» $\mathrm{y}$, particularmente la cultura (científica - formal o fáctica-, tecnológica, política, social, económica, comunicativa, ética, estética y hasta emotiva) desu época, ofrece particulares dificultades a las ciencias humanas.

A diferencia de lo que ocurre con las ciencias de la naturaleza, las ciencias sociales presentan claramente una menor base consensual y una menor homogeneidad en sus micro y macrovisiones del mundo. Es en este sentido en el que la afirmación empírica acerca de la presencia de relaciones de valor en el origen de las hipótesis sociológicas se presenta como específicamente problemática. Las distintas cosmovisiones generan una diversidad de «puntos de vista» que puede propiciar (al menos en principio) un pluralismo teórico incontrolado. Y la desagregación teórica, por supuesto, no parece precisamente el camino más acertado hacia la ciencia.

El problema radica precisamente en la multiplicidad de «puntos de vista» posibles que pueden operar como criterio heurístico de selección de problemas. A ello ya apuntaba claramente Max Weber señalando cómo

«Meaning and Scientific chage», donde presenta críticamente las posiciones de Kuhn y Feyerabend, pp. 58-101 en su Reason and the searcb for knowledge. Investigations in the philosopby of science, Reidel Publ. Co., 1984.

24. Kuhn T. S., 1962 (inglés), La estructura de lạs revoluciones científicas, Fondo de Cultura Económica, México, 1971, 1; ed.

25. Giner, $S$, «E1 progreso de la conciencia sociológica», pp. 7-29, en Sistema, núm. 1, enero 1973, p. 21. 
las «individuaciones» o delimitaciones desde las vatias «orientaciones de valor» admiten la diferencia de ángulos de apreciación o distintos «puntos de abordaje» posibles de valoración (1906: 131-132). Su admisión, por consiguiente, fundamenta el pluralismo teórico de la sociología (p. 145).

Weber extiende explícitamente la crítica lógica a los valores en tanto -objeto de las ciencias de la cultura, pero su función en relación con los juicios de valor como medios de conocimiento queda críticamente irresuelta. Su posición es expuesta en la polémica con E. Meyer (1906: 111): «El análisis causal to proporciona absolutamente ningún juicio de valor, y el juicio de valor no es, en absoluto, una explicación causal.» $\mathrm{Y}$ continúa elucidando la tesis más adelante: «Lo cual no significa, por cierto, que la consideración causal de su génesis no pueda resultar esencial para posibilitar "psicológicamente" la "comprensión" de la significación de un valor" (ibid., n. 6). Mientras, según esta afirmación, el juicio en torno a la validez de un valor, como ya se ha expresado, no es asunto de la crítica técnica, los juicios de valor us uorientaciones de valor» se encuentran en el origen de las hipótesis científicas, pero escapan a la crítica. Tales puntos de partida se presentan como irrefutables fácticamente. En la propuesta weberíana los presupuestos últimos de valor escapan al análisis crítico y quedan inmunizados contra los criterios de contrastación, contrafacticidad, comparabilidad y eficacia, por ejemplo. Operan, al modo axiomático, como principios indemostrables que el mismo Weber tiene la tentación de elevar a la categoría de convicciones de $f e^{26}$ entre las que hay que optar sin recurtir a la ciencia (1906: 131-132).

Su solución crítica en torno al problema de los valores es, por lo tanto, parcial. Ello queda bien expresado en (1906: 104): «Sóto limitando y resolviendo problemas concretos se fundaron las ciencias, y sólo así desarrollaron su método (...) cuando a consecuencia de desplazamientos notables de "puntos de vista", a partir de los cuales cierta materia se vuelve objeto de exposición, surge la idea de que esos "puntos de vista" nuevos exigen también una tevisión de las formas lógicas dentro de las cuales se movía el "cultivo" consagrado, lo que arroja incertidumbre sobte la "esencia" de la propia labor.» No obstante, en la resolución del problema de los presu-

26. Con la evidencia historica de la existencia de distintas tomas de posición (no hay que olvidar que Weber configura su posición dentro de las controversias de la Verein für Sazialpolitik: cf. Dabrendort, 1961 [aiemán], "Values and Social Sciences", cap. I en sus Essays in the Theory of Society, Londres, RKP, 1968), Weber no podía encontrar «niguna autoridad zacional» a la que pudiera teferirse para legitimar la suya como la única posición razonable (cf. el comentario de Albert, 1968, p. 106 y, en general, todo el tratamiento del tema que hace en «EI modelo clásico de la racionalidad y la discusión sobre el valot», III, pp. 102 y ss.). 
Ciencia y valotes: un reordenamiento de argumentos a favor de la ciencia social

puestos últimos de valor, no cabe duda de que Weber opta por la «certidumbre». Su solución al problema de la heterogeneidad y multiplicidad de «puntos de vista» orientados a los valores (lo que él llama la «dimensión subjetiva» de las ciencias de la cultura: $i b i d$.) acaba en la aceptación de su inmunidad crítica. Cerrados a la relativización crítica, estos presupuestos últimos de valor se tornan irracionales. Ello le ha valido lecturas diferenciadas de positivistas y hermenéuticos y extendido su ámbito de influen$\mathrm{cia}^{27}$ no obstante, también ha constituido uno de sus flancos más sensibles a la crítica. ${ }^{28}$

La historia de la ciencia señala reiteradamente cómo la coexistencia de varias teorizaciones diferenciadas sobre un único objeto no es ella misma problemática, al menos no cuando puede tesolverse críticamente. El probiema central, pues, en ei planteamiento weberiano - digámoslo de nuevo- es que, mientras extiende la crítica lógica a los juicios (valotativos) de hecho, inmuniza de la misma tales juicios en tanto puntos de partida o medios de conocimiento.

El pluralismo incontrolado en sociología viene reforzado por lo que Amartya Sen (1981) denomina la multiplicidad de «intereses de uso» in. formativo de los diagnósticos, pronósticos, previsiones, descripcionies o teorizaciones en general de la sociología moderna. A diferencia de lo que ocurre en las ciencias naturales, que en relación al interés informativo de sus tesis fácticas alcanzan una gran homogeneidad, las ciencias sociales no logran la uniformidad de sus pautas y evaluaciones (sus propios estándates) y quedan a merced de la demanda. La no-uniformidad de intereses informativos, que en si misma no es incompatible con la objetividad de las aserciones fácticas, se sustenta sólo parcialmente en el problema anterior puesto que tiene su propia pragmática. No obstante, vale considerar que tal multiplicidad de intereses informativos ha operado como mecanismo de feed back $y$ ha permitido «funcionar» a la sociología sin acabar de resolver sus problemas de fondo. De aquí quizá la sobreptoducción sociográfica y la persistente tendencia a la «regionalización» de las microteorías, indiscriminadas y subvencionadas, de las que habla $\mathrm{L}$. S. Warshay ${ }^{29}$ refiriéndose a la sociología notteameticana (o no).

27. Ibid.

28. Para una revisión de las distintas críticas a $\mathrm{M}$. Webet, cf. Guenther Roth, «Political critiques of Max Weber. Some implications for political sociology», en American Sociological Review, vol. 30, 1965, pp. 213 y ss.

29. Warshay, L, H., The current state of sociological theory: A critical interpretation, N.Y., McKay, 1975.

Todas las citas de Max Weber corresponden a la edición castellana de Amorrortu, B. A., 1973, 1978: Ensayos sobre metodología sociologica. 
«Papers»: Revista de Sociologia

Se puede observar, sin embargo, que ambos problemas, aunque vinculados a la naturaleza específica del objeto sociológico, tienen más bien relación con la no-propiedad o no-resolución de criterios epistémicos y heu rísticos congruentes con el análisis crítico y con la emergencia de la decisión en el campo del conocimiento y de la praxis científica. En ninguno de los dos casos sirven realmente para argumentar contra la objetividad de los resultados cognitivo-informativos de la crítica técnica aplicada a valores. En todo caso, estos problemas señalan su límite de desarrollo y el escollo adicional de su inmediata operatividad práctica.

Bellaterra, noviembre de 1988 